\title{
A P300-Based Brain-Computer Interface for Chinese text Input
}

\author{
Yang $\mathrm{Yu}^{\mathrm{a}}$, Zongtan $\mathrm{Zhou}^{\mathrm{b}}$, Jingsheng Tang ${ }^{\mathrm{c}}$, Peng $\mathrm{Du}^{\mathrm{d}}$, Yadong Liu ${ }^{\mathrm{e}}$, and \\ Dewen $\mathrm{Hu}^{\mathrm{f}^{\prime}}$
}

College of Mechatronic Engineering and Automation, National University of Defense Technology, Changsha, Hunan, 410073, China

ayuyangnudt@hotmail.com, bnarcz@163.com, '81089979@qq.com, ddupeng13579@163.com, eliuyadong1977@163.com, ${ }^{*}$ dwhu@nudt.edu.cn

Keywords: Brain-Computer interface (BCI), Chinese speller, P300, Electroencephalography

\begin{abstract}
In this study, a P300 visual spelling system was presented using a novel method to input Chinese sinograms developed on a Hanyu Pinyin-based method. This method transcribes a Chinese pinyin into an initial consonant and vowel component according to its mandarin pronunciation. In this paradigm, each sinogram was input by selecting the initial consonant followed by the vowel components, and then by selection of the sinogram itself. Five healthy participants participated in the study and achieved an average offline accuracy of $90.2 \%$ and an average online input speed of one sinogram per 69 seconds. The experiment results make P300-BCIs accessible to individuals whose language has a logographic writing system.
\end{abstract}

\section{Introduction}

Brain-computer interfaces (BCIs) are communication and control pathways that directly translate brain activities into computer control signals, garnering a growing interest in recent decades [1,2]. A prevalent form of BCIs is the P300 BCI, which uses non-invasive electroencephalography (EEG) to recognize the $\mathrm{P} 300$ response of an attended stimulus. The P300 is an event-related potential elicited by a rare or "oddball" target stimulus, and it is often recorded over the parietal cortex approximately $300 \mathrm{~ms}$ after the presentation of the stimulus [3]. P300 BCI systems are mainly used as spelling devices because they are superior to other types of BCIs for this application [4].

P300 potential has been widely used in BCIs for spelling applications. The classic P300-based matrix speller, originally presented by Farwell and Donchin [5], allows a user to communicate one of the 36 symbols presented in a $6 \times 6$ matrix. Based on this classic paradigm, numerous adjustments to the speller have been proposed to improve the speed and accuracy of the P300 speller. Most of those previous BCI systems have mainly focused on languages that are written in alphabetic script, such as English and German [6-10]. However, Chinese hanzi (or sinograms) has more than 11,000 commonly used characters. Such languages with logographic writing systems pose a significant challenge to P300-BCI systems because only a handful of the thousands of distinct logograms in the writing system can be simultaneously presented to the user at any moment. A primary method for Chinese text input is based on Hanyu Pinyin [11], or Pinyin, employs the 26 letters of the Roman alphabet to represent the entire inventory of Putonghua pronunciation. In the current study, we developed a P300 visual speller for sinograms input using Pinyin-based method. This was done by entering the sequence of letters that corresponds to its pronunciation.

\section{Materials and Methods}

Subjects. Five native Chinese speakers participated in this study (male, aged 23-27 years, with mean age and std $26.43 \pm 2.12$ years). All of the subjects were able-bodied and without any known cognitive deficits. Three out of the five participants had previous BCI experience, and the other subject was complete novices. All participants provided written informed consent for the procedure after receiving a complete description of this study. 
Data collection and signal processing. The P300 ERP was recorded using an EEG cap embedded with 5 electrodes covering $\mathrm{Cz}, \mathrm{Pz}, \mathrm{P} 3, \mathrm{P} 4$, and $\mathrm{Oz}$ (Fig. 1), which have previously been shown to contribute most to classification accuracy 24 , based on the 64-channel modified international 10-20 system. Each channel was referenced to the right mastoid with the forehead as the ground. The electrode impedance was maintained below $5 \mathrm{k} \Omega$. The EEG signals were amplified using a BrainAmp DC Amplifier, and were digitized at $250 \mathrm{~Hz}$, and filtered using a $50 \mathrm{~Hz}$ notch filter. The stimulus presentation, data collection, offline signal processing, and experimental procedures were conducted using the BCI2000 framework [12], which provides a Python interface for stimuluspresentation and a Matlab interface for signal processing.

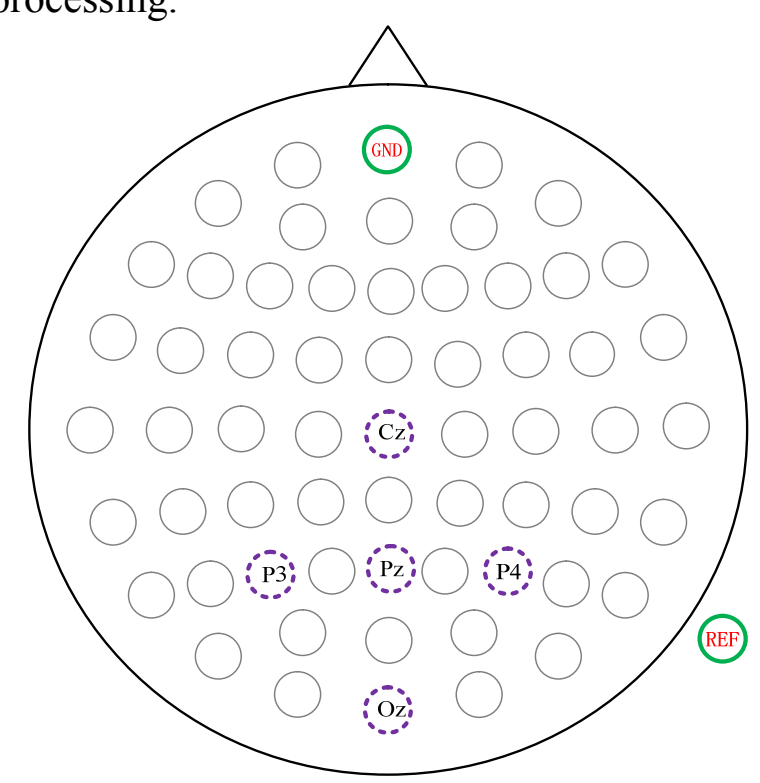

Fig. 1. Electrode set for recording and analysis. The head is viewed from above and the nose points to the top of the page.

Experimental procedure. The subjects sat in an unshielded room, approximately $1 \mathrm{~m}$ in front of a 22' 'LED monitor that displayed the experimental stimuli. The design and purpose of the speller were explained to each subject in detail, and a short familiarization session was performed prior to the initiation of the experiments. The experiment consisted of two parts: calibration followed by online testing, and each participant completed the experiment in three successive days.

The experiment paradigm for the calibration experiments is shown in Figure 3A. Two of the ten flashes contain the target. Each subject participated in 3 sessions, and each session consisted of 10 runs. In each run, one target character flashed for 8 trials, where one trial is defined as a complete cycle of flashes in which all of the rows and columns flash once. Each intensification was for $120 \mathrm{~ms}$ followed by an $80 \mathrm{~ms}$ interstimulus interval during which time no stimulus was intensified. For each intensification, an $800 \mathrm{~ms}$ segment of EEG data was extracted from each channel for use in the classification (see Figure 2 for details).

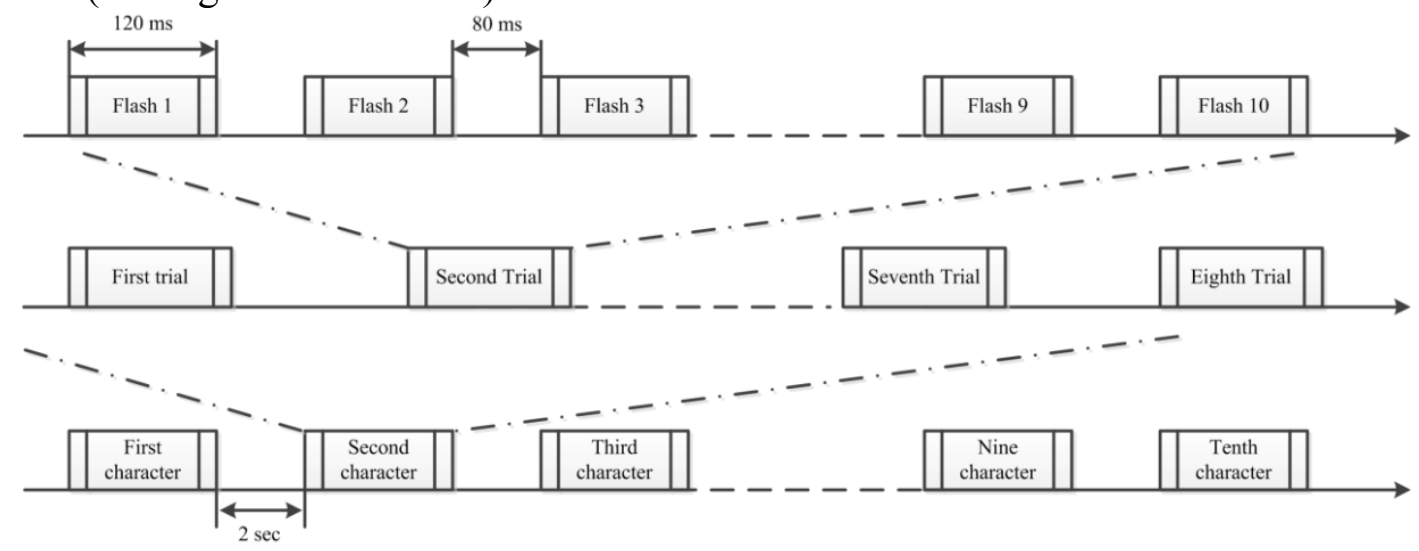

Fig. 2. Time course of the flashes. 
During the online testing experiment, the subject was required to input ten previously selected characters one by one based our Pinyin-based method. This was done by entering the sequence of letters that corresponds to its pronunciation. (see Figure 3 for details). There was a five minute break between each session to allow the participant to rest. The high-frequency sinograms were prior presented to assist the user to locate target more easily. PgUp (page up) and PgDn (page down) were designed to allow the participant to turn to the page where the expected target was located.
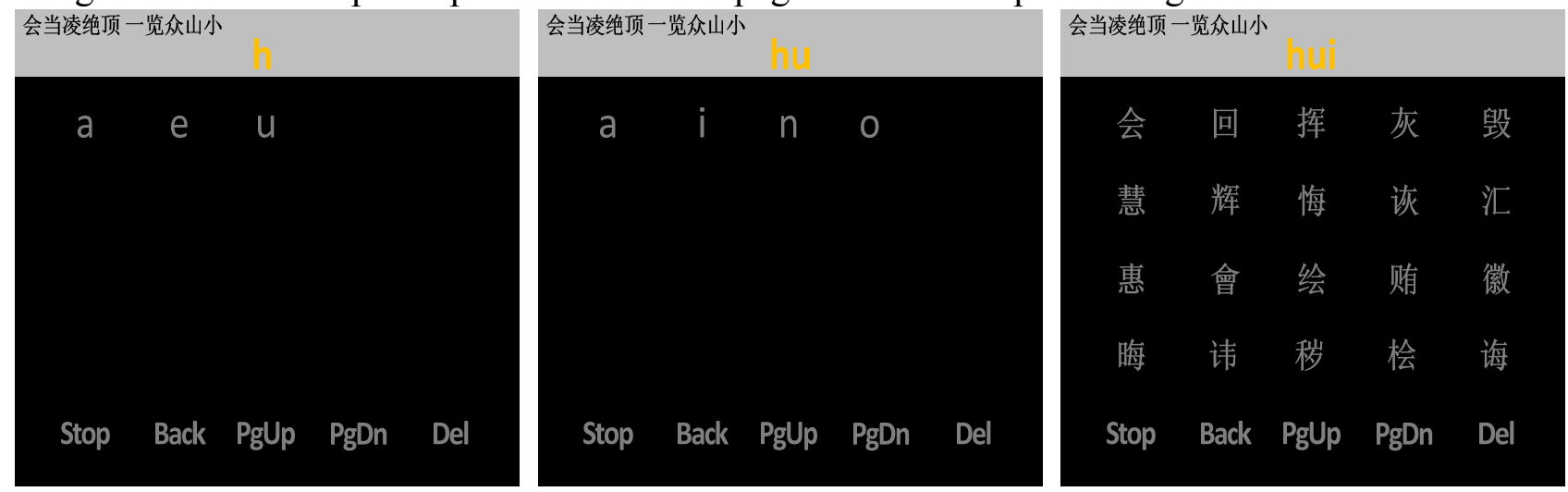

Fig. 3. Illustration of the spelling procedure. Left: The stimulus paradigm after the initial letter was selected. Right: The stimulus paradigm after the last letter was inputted.

Classification and evaluation. The presence or absence of the target P300 from the chosen EEG features is a binary classification problem. In this study, we used step-wise linear discriminant analysis (SWLDA [13]) to solve this classification problem [14, 15]. Calibration of the classifier coefficients was performed offline using the data collected in the calibration experiments. The performance of the speller was evaluated using all the data from both the calibration and the online experiments. During the calibration experiments, the classification accuracy was calculated as the percentage of characters that were correctly inputted, and the information transfer rate (ITR) was used to evaluate the BCI system. During the online experiment, we calculated the communication speed as the average time to input one sinogram.

\section{Results and conclusion}

Each subject participated three sessions of the calibration experiment, and each session consisted of 10 runs, with each run consisting of 8 trials. The offline classification accuracy and the ITR achieved when each participant used two trials per selection are illustrated in Table 1.

During the online sinogram-input experiments, each participant was instructed to input ten sinograms. Table 1 shows the sinogram input performance obtained by each of participants at 2 trials per selection during online testing. When permitted to correct errors, the participants achieved a mean input speed of one sinogram per $69 \mathrm{~s}$.

Table 1. The offline accuracy and online communication speed obtained by each subject.

\begin{tabular}{|c|c|c|c|}
\hline \multirow{2}{*}{ Participant } & \multicolumn{3}{|c|}{ Two trials per selection } \\
\cline { 2 - 4 } & Offline accuracy & ITR & Online speed (times/per sinogram) \\
\hline S1 & $93.00 \%$ & 40.44 & $65 \mathrm{~s}$ \\
\hline S2 & $93.70 \%$ & 41.83 & $64 \mathrm{~s}$ \\
\hline S3 & $91.00 \%$ & 38.28 & $68 \mathrm{~s}$ \\
\hline S4 & $84.50 \%$ & 34.27 & $78 \mathrm{~s}$ \\
\hline S5 & $89.00 \%$ & 36.16 & $70 \mathrm{~s}$ \\
\hline Mean & $90.20 \%$ & 38.1 & $69 \mathrm{~s}$ \\
\hline
\end{tabular}




\section{Acknowledgements}

This work is supported by the National Natural Science Foundation of China (Grant No 91320202, and 91420302). The National Basic Research Program（Grant No 2015CB351706)

\section{References}

[1] T. Y. Yu, Y. Q. Li, J. Y. Long, Z. H. Gu, Surfing the internet with a BCI mouse, J Neural Eng 9 (2012), 036012.

[2] D. Zhang, A. Maye, X. R. Gao, B. Hong, A. K. Engel, S. K. Gao, An independent brain-computer interface using covert non-spatial visual selective attention, J. Neural Eng 7 (2010), 016010 .

[3] M. Fabiani, G. Gratton, D. Karis, E. Donchin, Definition, identification, and reliability of measurement of the P300 component of the event related potential, Advances in Psychophysiology 2 (1987), 1-78.

[4] C. Guger, S. Daban, E. Sellers, C. Holzner, G. Krausz, R. Carabalona, F. Gramatica, G. Edlinger, How many people are able to control a P300-based brain-computer interface (BCI)?, Neurosci Lett 462 (2009), 94-98.

[5] L. Farwell, E. Donchin, Electroencephalogr Talking off the top of your head: toward a mental prosthesis utilizing event-related brain potentials, Clin Neurophysiol 70 (1988), 510-523.

[6] D. Krusienski, E. Sellers, F. Cabestaing, S. Bayoudh, D. McFarland, T. Vaughan, J. Wolpaw, A comparison of classifi-cation techniques for the P300 Speller, J Neural Eng 3 (2006), 299-305.

[7] G. Townsend, B. LaPallo, C. Boulay, D. Krusienski, G. Frye, C. Hauser, N. Schwartz, T. Vaughan, J. Wolpaw, E. Sellers, A novel P300-based brain-computer interface stimulus presentation paradigm: Moving beyond rows and columns, Clin Neurophysiol 121 (2010), 1109-1120.

[8] G. Frye, C. Hauser, G. Townsend, E. Sellers, Suppressing flashes of items surrounding targets during calibration of a P300-based brain-computer interface improves performance, J Neural Eng 8 (2011), 025024.

[9] Y. Li, C. S. Nam, B. B. Shadden, S. L. Johnson, A P300-based brain-computer interface: Effects of interface type and screen size, International Journal of Human-Computer Interaction 27 (2010), $52-68$.

[10]E. W. Sellers, D. J. Krusienski, D. J. McFarland, T. M. Vaughan, J. R. Wolpaw, A P300 event-related potential brain-computer interface $(\mathrm{BCI})$ : The effects of matrix size and inter stimulus interval on performance, Biol Psychol 73 (2006), 242-252.

[11]B. Yin, M. Felley, Chinese Romanization. Pronunciation and orthography, China: Beijing, 1990

[12] Schalk G, McFarland DJ, Hinterberger T, Birbaumer N, Wolpaw JR. BCI2000: a general-purpose brain-computer interface (BCI) system. IEEE Trans Biomed Eng 2004 (51):103443.

[13] N. R. Draper, H. Smith, Applied regression analysis (2nd ed.), New York, 1981.

[14] D. J. Krusienski, E. W. Sellers, D. J. McFarland, T. M. Vaughan, J. R. Wolpaw, Toward enhanced P300 speller perfor-mance, J Neurosci Methods 167 (2008), 15-21.

[15]D. B. Ryan, G. E. Frye, G. Townsend, D. R. Berry, S. Mesa-G., N. A. Gates, E. W. Sellers, Predictive spelling with a P300-based braincomputer interface: Increasing the rate of communication, International Journal of Human-Computer Interaction 27 (2011), 69-84. 